\title{
Rivaroxaban does not impair fracture healing in a rat femur fracture model: an experimental study
}

\author{
Tim Klüter ${ }^{1{ }^{*}+}$, Matthias Weuster ${ }^{1 \dagger}$, Stefan Brüggemann ${ }^{1}$, Leif Menzdorf ${ }^{1}$, Stefanie Fitschen- Oestern ${ }^{1}$, \\ Nadine Steubesand ${ }^{1}$, Yahya Acil ${ }^{3}$, Thomas Pufe ${ }^{2}$, Deike Varoga' ${ }^{1}$, Andreas Seekamp ${ }^{1}$ and Sebastian Lippross ${ }^{1}$
}

\begin{abstract}
Background: The prescription of the oral anticoagulant rivaroxaban to prevent thromboembolic episodes associated with orthopaedic surgery has dramatically increased since it was introduced. Rivaroxaban is beeing prescribed although recent in-vitro studies revealed that it impaired osteoblast metabolism. In this study we analysed the effect of rivaroxaban on fracture healing in a rat femur fracture model.
\end{abstract}

Methods: Femur fractures were created by a 3-point-bending device in 48 Wistar rats and subsequently stabilized by intramedullary nailing. After the surgical procedure animals were randomised into four groups. Two groups were fed with $3 \mathrm{mg}$ rivaroxaban per $\mathrm{kg}$ body weight per day and two control groups were fed with chow only. Animals were euthanized 28 or 49 days after surgical procedure. Femurs underwent undecalcified histologic staining micro CT scanning and biomechanical testing. The statistical significance was evaluated using one-way Anova with Bonferroni correction.

Results: Micro CT-scans revealed significantly increased volume of bone tissue in the fracture zone between day 28 and 49. During the same time callus volume decreased significantly. Comparing the fracture zone of the rivaroxaban group to the control group the treated group revealed a larger callus and a marginal increase of the tissue mineral density. The torsional rigidity was not influenced by the treatment of rivaroxaban.

Conclusion: In the present study we were able to demonstrate that rivaroxaban does not impair fracture healing in a rat femur fracture model. Considering the fact that low molecular weight heparins delay fracture healing significantly, rivaroxaban might be an improved alternative.

\section{Background}

Thrombembolic complications constitute a main cause of mortality after fractures of the lower limb. Several studies document that without thromboprophylaxis the incidence of venous thromboembolism (VTE) after hip fracture, total hip arthoplasty or multiple trauma goes up to one third to one half $[1,2]$. Thromboprophylaxis using heparin can reduce thrombembolic complications by up to 40 to $60 \%$ [3]. During the last decades low molecular weight (LMW) heparins replaced unfractionated heparin (UH) and became gold standard for thromboprophylaxis in orthopaedic surgery [4].

\footnotetext{
* Correspondence: tim.klueter@uksh.de

${ }^{\dagger}$ Equal contributors

'Department of Trauma Surgery, University Medical Center of

Schleswig-Holstein, Campus Kiel, Germany

Full list of author information is available at the end of the article
}

In 1956 Stinchfield and colleagues documented the effects of heparin and warfarin on bone repair. They described that daily administration of heparin causes attenuated fracture healing in rabbit and canine models [5]. Street et al. analyzed the effect of LMW heparin on fracture healing of the rabbit. Histomorphometric, histologic and immunohistochemical testings demonstrated that the bone repair was attenuated at all times in animals receiving Enoxaparin [6]. Besides LMW heparins have been shown to cause osteoporosis when given for longer than 8 weeks [7]. In case of fractures, pericytes migrate from peristostal vasculation and transform into ostogenic progenitor cells. UH and LMW heparin bind to endothelial cells and osteoblast, reduce their activity and prohibit neovascularisation [8]. On the other hand decreased blood clotting due to an enlarged fracture hematoma has a negative influence on the initial phase of the fracture healing. Potassium channels in osteoblasts 
and endothelial cells are influenced negatively by hyperkalaemia, by enabling them to react on proliferative cytokines. This leads to significant cytostasis and cell distraction in the fresh hematoma [6,9]. Both mechanisms, the binding of endothelial cells and osteoblasts as well as the bleeding tendency may cause a delay of fracture healing.

Recently, the novel oral anticoagulant rivaroxaban $\left(\right.$ Xarelto $^{\odot}$ ) was developed to safely and effectively prevent VTE. Rivaroxaban inhibits coagulation factor Xa directly by binding to its active centre. Factor $\mathrm{Xa}$ is the active form of the coagulation factor thrombokinase and is synthesised in liver. Long et al. have recently shown that in comparison with LMW heparins rivaroxaban reduced the incidence of VTE by $45 \%$ without increasing the risk of bleeding in patients with lower extremity fractures [10]. Patients receiving rivaroxaban do not need to undergo daily injection or regular drug monitoring and therefore profit from a higher comfort and greater compliance. During the last years oral factor Xa inhibitors gained continuously more popularity in thromboprophylaxis after total hip and knee arthroplasty. It is assumed that oral factor Xa inhibitors will be established in thromboprophylaxis of trauma patients soon.

So far the effect of rivaroxaban on fracture healing has not been investigated. In in vitro studies Gigi et al. have recently revealed an inhibition of osteoblast metabolism caused by rivaroxaban [11]. Considering these facts the authors hypothesized that thromboprophylaxis with this new substance would promote interfragmentary hematoma collection and delay bone formation during fracture healing. Therefore we investigated the effect of rivaroxaban in an established rodent fracture model, first published by Bonnarens [12]. The application and dose is well investigated because first developments and tests were conducted in rats [13].

\section{Methods}

\section{Animals}

Ethical approval was obtained from the regional Ethics committee Schleswig-Holstein, Germany (V 312-72241.121-9 (8-1/09)). 48 female Wistar-rats weighing $230 \pm 30$ g were obtained from the local Service Unit of the authors' institution. The animals were kept two per cage with free access to rat-chow and water. The lighting was maintained on a 12-hour light-dark cycle. Analgesic treatment was administered to all animals 4 times a day via drinking water $\left(100 \mathrm{mg} / \mathrm{kg}\right.$ metamizole sodium (Novalgin ${ }^{\circ}$, Hoechst, Unterschleißheim, Germany)). After the surgical procedure four groups were randomised, two groups were treated with $3 \mathrm{mg}$ rivaroxaban per $\mathrm{kg}$ body weight per day together with their chow, two control groups were only fed with chow (Table 1). The animals were euthanized on day 28 or 49 after surgical procedure.
Table 1 Group distribution of 48 rats

\begin{tabular}{lll}
\hline Time point & Test & Quantity \\
\hline Day 28 & Biomechanical testing & 6 rats each group \\
& Micro CT analysis & 6 rats each group \\
& Histologic assessment & \\
Day 49 & Biomechanical testing & 6 rats each group \\
& Micro CT analysis & 6 rats each group \\
& Histologic assessment & \\
\hline
\end{tabular}

\section{Surgical procedure}

Implants and surgical equipment were sterilized in an autoclave. Sterile gowns, gloves, surgical mask and theatre caps were used. Preoperatively each rat received $0.06 \mathrm{mg}$ buprenorphine orally. The animals were anaesthetized with Fentanyl $0.005 \mathrm{mg} / \mathrm{kg}$, Midazolam $2.0 \mathrm{mg} / \mathrm{kg}$ and Medetomedine $0.15 \mathrm{mg} / \mathrm{kg}$ by intraperitoneal injections. The rat leg was shaved and washed with chlor-hexidine. Medial to the patella the skin was incised and the patella dislocated to the lateral side, so that the condyles were exposed. A 0.90x40 mm sterile cannula (Braun, Melsungen, Germany) was drilled though the medullary channel using an intercondylar approach. The sharp end was place to the proximal end of the femur and the rest of the cannula was cut off, so that no end extended beyond the bone. The patella was repositioned over the knee joint and the skin was closed with resorbable thread. A closed middiaphyseal transverse fracture was created in the right femur by three-point bending as described by Bonnarens [12] Anaesthesia was antagonized by intraperitoneal Naloxon $0.12 \mathrm{mg} / \mathrm{kg}$, Flumazenil $0.2 \mathrm{mg} / \mathrm{kg}$ und Atipamezol $0.75 \mathrm{mg} / \mathrm{kg}$. Animals were sacrificed by $\mathrm{CO}_{2}$ asphyxation on day 28 and day 49 . Bones were dissected, fixed in $4 \%$ paraformaldehyde and stored in $70 \%$ Ethanol or used for biomechanical testing.

\section{Biomechanical testing}

Torsional rigidity was analysed as described by Manigrasso [14]. Testing was performed using a custom built torsional servohydraulic testing device as described by Turner et al. and Engesaeter et al. $[15,16]$ using a $20 \mathrm{Nm}$ reaction torque load cell. Femurs were tested to failure at an actuator head displacement rate of $1 \%$ second. The peak torque and angle at failure were measured from the torque-angle deflection curves.

\section{Micro CT analysis}

For a detailed qualitative and quantitative 3-D evaluation, the fracture site was examined in a micro CT scanner (Scanco Medical AG, Bassersdorf, Switzerland) equipped with a $70 \mu \mathrm{m}$ focal spot microfocus X-ray tube as a source. During scanning, the femoral bones were placed in phosphate buffered saline. Radiographic projections were performed at $70 \mathrm{kV}$ and $114 \mu \mathrm{A}$ with a fixed integration time 


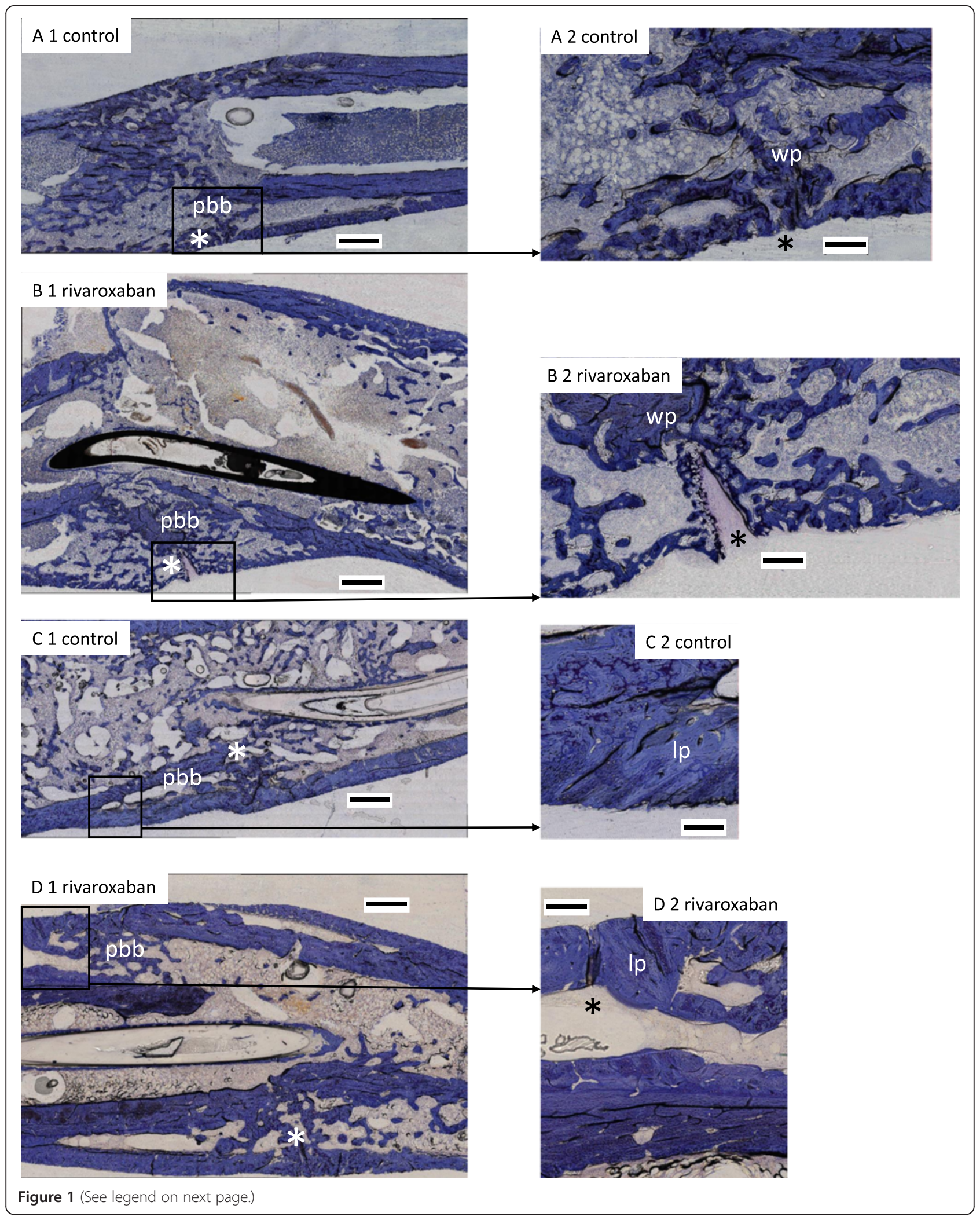


(See figure on previous page.)

Figure 1 Photomicrography of fracture callus from rat femur. Panoramic views (1) and high magnification (20x) photomicrography (2) of fracture healing and callus formation was illustrated using calcified bone histology technique with toluidine blue staining. The magnification points up the remodelling zone within the fracture gap. 28 days after fracture the fracture gap $\left(^{*}\right)$ and a sufficient callus is noticeable with partial bony bridging $(A+B) .49$ days after fracture the femurs demonstrate increased bone tissue with lamellar bone $(C+D)$. No significant difference could be seen between control and treatment group. Bar represents 1 mm (A1 - D1) or $100 \mu m(\mathbf{A} 2-\mathbf{D} 2)$ respectively. pbb = partial bony bridging; l $p=$ lamellar part; $w p=$ woven part.

of $200 \mathrm{~ms}$. For image acquisition, the specimen was mounted on a turntable shifted automatically in an axial direction over $180^{\circ}$, taking 500 projections. Threedimensional images were stored in 3-D arrays with an isotropic voxel size of $16 \mu \mathrm{m}$ and a pixel size of $1024 \times 1024$. The region of interest (ROI) covered a total range of $8.4 \mathrm{~mm}$ (encompassing $4.2 \mathrm{~mm}$ proximal and distal to the fracture line). For quantitative analysis of bone formation within the ROI Scano Evaluation software (Scano Medical, Brüttisellen, Switzerland) was used to obtain the volume of interest. Depending on the grey threshold these volumes were reflected to highly mineralised tissue (bone, 33 to $60 \%$ ) and low mineralised tissue (callus, 17.5 to $33 \%$ ). Tissue mineralized density was calculated by dividing the volume of the mineralised callus by the tissue's density. Due to technical problems the results of 3 rats treated for 49 days were deleted somehow. When this error was noticed, samples could not be scanned again.

\section{Preparation of specimens}

To illustrate the micro-architecture of the callus, undecalcified bone histology was performed. The bones were fixed in $10 \%$ neutral buffered formalin for 14 days at room temperature, dehydrated with ascending concentration of ethanol and embedded in methylmetacrylate for 5 days at $4^{\circ} \mathrm{C}$. Afterwards tissue was soaked in methylmethacrylat monomer, nonylphenyl-polyethyleneglycol acetate and azoisobutyronitrile (all Sigma-Aldrich, St. Louis, USA). The blocks were released from the glass vials and undecalcified sections of $40 \mu \mathrm{m}$ were sawed and grinded using an EXAKT diamond saw system (Exakt, Norderstedt, Germany).

\section{Histologic assessment}

For histologic staining sections were lubricated with $0.1 \%$ formic acid for 2 minutes and washed with aqua afterwards. The samples were immersed in $20 \%$ methanol for 1 hour and stained with toluidine blue or hematoxylin and eosin.

\section{Statistical analysis}

Statistical analysis was performed using a standard software application (SPSS Inc., Chicago, IL, USA). Results of CT scanning and torsional testing were expressed as the means \pm standard deviation of tested groups. The different groups were tested for normality using Kolmogorov-
Smirnov test. Statistical significance was evaluated using one-way Anova with Bonferroni correction. Sample distribution for torsional testing and CT scanning were illustrated with Box and whisker plots.

\section{Results}

All animals recovered quickly after the surgical procedures and fully mobilized within two days. No signs of pain or dysfunction in the physical motion was recognized. No animal had to be excluded because of death, implant dislocation or soft tissue infection.

\section{Histology}

For assessment of fracture healing and callus formation we used a standard calcified bone histology technique. Thereby we could illustrate the cellular and mineralised components of the callus in the longitudinal axis, which provides vital information on bone turnover or bone formation and resorption. 28 days after closed femur fracture the fracture gap was still visible and sufficient callus had arisen (Figure 1). Tolouidine blue staining showed partial bony bridging of the gap in the 28 days group and the bridging increased 49 days after fracture. During

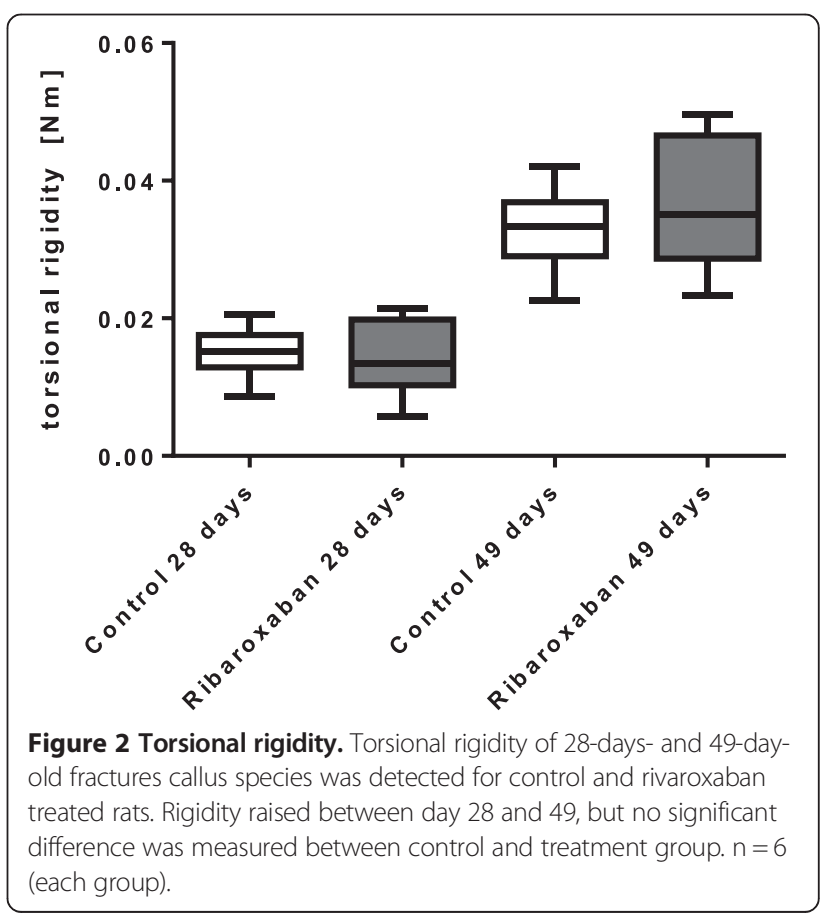


the remodelling processes of the endosteal bony callus the amounts of newly formed bone increased between day 28 and 49. Bony callus consists of woven and lamellar bone, and here the lamellar part increased and the woven part decreased.

No significant difference in fracture healing could be identified in either comparison group after 28 or 49 days. Over all the callus sizes showed great variability amongst all groups.

\section{Biomechanical analysis}

The operated femurs were analysed for torsional rigidity 28 and 49 days after the surgical procedure. Parameters such as torsional rigidity calculated from torsion tests are convenient for determining the structural and material properties of the healing tissue. After 4 weeks of rivaroxaban diet the femur callus showed no significant difference in torsional rigidity compared to the control group $\left(0.0154 \pm 0.0039 \mathrm{~N} / \mathrm{m}^{2}\right.$ versus $0.0141 \pm 0.0056 \mathrm{~N} / \mathrm{m}^{2}$; $\mathrm{p}=0.81$ ). Even after 7 weeks of rivaroxaban treatment rigidity did not change significantly (Figure 2 ).

\section{Micro CT scan of interfragmentary zone}

The callus volume was determined from $4.2 \mathrm{~mm}$ proximal to $4.2 \mathrm{~mm}$ distal of the fracture gap by micro CT scanning. During fracture healing bone tissue increaed in rivaroxaban treated rats from $65.9 \pm 8.1 \mathrm{~mm}^{3}$ to $89.2 \pm 2.7 \mathrm{~mm}^{3}$ $(\mathrm{p}=0.06)$, whereas callus volume decreased from $42.17 \pm$ $5.3 \mathrm{~mm}^{3}$ to $9.7 \pm 3.1 \mathrm{~mm}^{3}(\mathrm{p}=0.04$; Figure 3$)$. After 28 days we detected an insignificant increase of callus






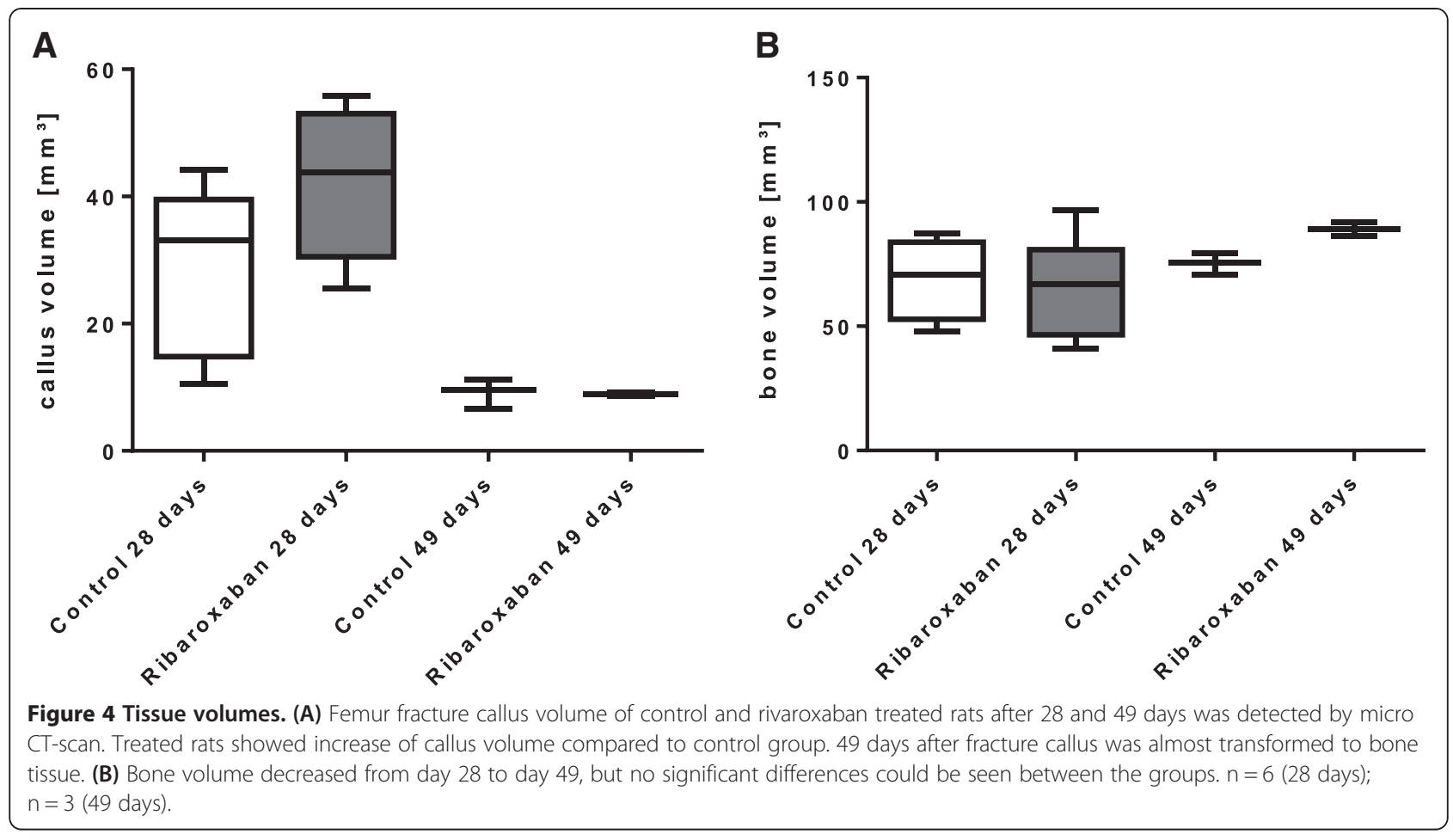

volume of specimens which underwent rivaroxaban treatment compared to the control animals $(28.37 \pm$ $13.42 \mathrm{~mm}^{3}$ versus $42.17 \pm 11.98 \mathrm{~mm}^{3}$; $\mathrm{p}=0.12$ ). After 49 days the callus was almost completely transformed to bony tissue, thus no significant alteration between both groups could be determined (Figure 4A).

No significant difference could be seen in bone volume between the groups at both points of time $\left(69.0 \mathrm{~mm}^{3} \pm\right.$ 17.0 versus $65.9 \mathrm{~mm}^{3} \pm 19.8$ and $75.2 \mathrm{~mm}^{3} \pm 4.3$ versus $89.15 \mathrm{~mm}^{3} \pm 3.9$ ) (Figure 4B).

\section{Tissue mineral density (TMD)}

To quantify the formation of the callus we analysed the TMD which was generated by micro CT scans within the defined area. After 28 days of rivaroxaban treatment we documented a marginal increase of the TMD compared to the control specimens $\left(0.057 \pm 0.017 \mathrm{mg} / \mathrm{cm}^{3}\right.$ versus $\left.0.055 \pm 0.012 \mathrm{~mm}^{3} ; \mathrm{p}=0.31\right)$. A similar trend was ascertained for 49 -day-old callus $\left(0.08 \pm 0.008 \mathrm{mg} / \mathrm{cm}^{3}\right.$ versus $\left.0.076 \pm 0.007 \mathrm{mg} / \mathrm{cm}^{3} ; \mathrm{p}=0.86\right)$, but both effects were statistically insignificant (Figure 5 ).

\section{Discussion}

Previous studies indicate a delay of callus remodelling due to thromboprophylaxis with UH and LMW heparin $[9,17]$. So far there is no study which investigated the effect of the new oral Factor Xa inhibitor rivaroxaban on fracture healing. Considering the fact that this anticoagulant is finding its way into treatment of trauma patients, it is indispensable to know its influence on the healing bone. Due to the complexity of interaction of the diverse cell types and tissues in vitro reproducibility of the bone healing process is not convincing. Therefore we utilised a classic established rodent fracture model [12].

In contrast to previous studies with heparins no delay of fracture healing relating to rivaroxaban treatment could be determined in the present study. Daily administration

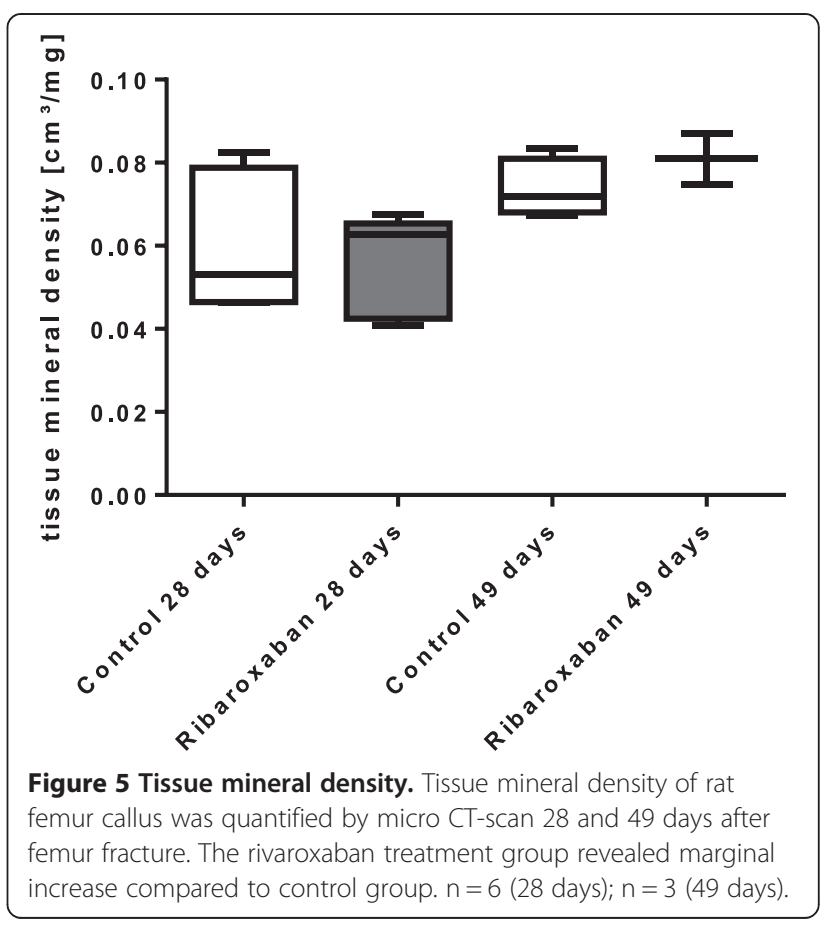


of the oral factor Xa inhibitor increased the callus volume insignificantly. To evaluate the progress of bone healing, we analysed callus by histological staining and documented a comparable repair process concerning the patterns of bone bridging, including periosteal, endosteal, intercortical patterns and mineralisation [18] We did not identify significant differences within the two groups at the same points of time. To quantify the callus mineralisation we performed micro CT scans as a valid assessment for bone stiffness. Previous studies revealed that TMD has the same validity for stiffness as the torsional righty [19-21]. The high resolution scan of the callus microstructure showed a small and not significant increase of TMD after rivaroxaban treatment. These results correlate with an in vitro study with cultured osteoblast which demonstrated a dose-dependent transient inhibition of osteoblast metabolism by rivaroxaban but no effect on mineralisation [11]. As another parameter for bone stiffness we measured the bone volume which did not increase significantly.

The torsional rigidity is a scientifically recognized standard to evaluate fracture healing $[6,20,22]$. Our study indicates that the torsional rigidity did not decrease after application of the oral anticoagulant. In contrast to that in previous studies LWM heparins result in a decrease of torsional stiffness and lower energy to fracture [6]. The authors explained this effect by binding of enoxaparin to vascular endothelium which disrupts callus vascular assembly and also by an increase of interfragmentary hematoma and subsequent cytotoxic effects on cells of the medullary callus. Due to technical problems we could not reach the minimal quantity to perform statistical analysis for the 49-days-old treatment group. Eventually the existing results could not justify further animal experimentation to reach statistical significance.

Taken together we could not document a negative effect in bone stiffness after rivaroxaban treatment. The higher callus volume on day 28 in combination with equal bone volume and tissue mineral density seems not to have an influence on the stability of the fracture.

\section{Conclusion}

In the present study we could for the first time demonstrate that thromboprophylaxis with rivaroxaban does not downgrade fracture healing in a rodent fracture model. Because of the low number of random samples, we are aware that this study is not powerful enough to give a general recommendation for the use of oral factor $\mathrm{Xa}$ inhibitor in case of trauma patients. Nevertheless considering the fact that LMW heparin, the gold standard for thromboprophylaxis, delays fracture healing significantly, rivaroxaban might be an improved alternative. The indications in prevention and treatment of thrombosis, particularly in trauma surgery, need further evaluation covering all aspects of bone repair.
Competing interests

The authors declare that they have no competing interests.

\section{Authors' contributions}

TK drafted the manuscript, participated in its design and performed the statistical analysis. MW carried out the surgical procedures and participated in its design and coordination. SB carried out the surgical procedures and the biomechanical testing and performed the statistical analysis. LM carried out the surgical procedures and carried out the biomechanical testing. SF conceived of the study and carried out the histologic assessment. NS carried out the histologic staining and participated in its design and coordination. YA carried out the histologic staining. TP participated in the design of the study and performed the statistical analysis. DV participated in the biomechanical testing. AS conceived of the study and participated in its design. SL designed the study, participated in its coordination and helped to draft the manuscript. All authors read and approved the final manuscript.

\section{Acknowledgements}

This work was supported by the AO-Foundation, by a grant from the Interdisciplinary Centre for Clinical Research (IZKF) within the faculty of Medicine at the RWTH Aachen University (T9-3; T9-5; T11-3) and in part by the Excellence Initiative of the German federal and state governments. We thank Dr Dophie Fargher at Starship Hospital, Auckland, NZL, for editing and proofreading the article.

\section{Author details}

${ }^{1}$ Department of Trauma Surgery, University Medical Center of Schleswig-Holstein, Campus Kiel, Germany. ${ }^{2}$ Department of Anatomy and Cell Biology, RWTH Aachen University, Aachen, Germany. ${ }^{3}$ Department of Maxillo-Facialy, University Medical Center of Schleswig-Holstein, Campus Kiel, Germany. ${ }^{4}$ Department of Trauma Surgery, University of Kiel,

Arnold-Heller-Strasse 3, 24105 Kiel, Germany.

Received: 7 September 2014 Accepted: 13 February 2015

Published online: 09 April 2015

\section{References}

1. Haake DA, Berkman SA. Venous thromboembolic disease after hip surgery: risk factors, prophylaxis, and diagnosis. Clin Orthop Relat Res. 1989;242:212-31

2. Salzman EW, Harris WH. Prevention of venous thromboembolism in orthopaedic patients. J Bone Joint Surg Am. 1976;58(7):903-13.

3. Handoll HH, Farrar MJ, McBirnie J, Tytherleigh-Strong G, Milne AA, Gillespie WJ. Heparin, low molecular weight heparin and physical methods for preventing deep vein thrombosis and pulmonary embolism following surgery for hip fractures. Cochrane Database Syst Rev. 2002:4:Cd000305.

4. Palmer AJ, Koppenhagen K, Kirchhof B, Weber U, Bergemann R. Efficacy and safety of low molecular weight heparin, unfractionated heparin and warfarin for thrombo-embolism prophylaxis in orthopaedic surgery: a meta-analysis of randomised clinical trials. Haemostasis. 1997;27(2):75-84.

5. Stinchfield FE, Sankaran B, Samilson R. The effect of anticoagulant therapy on bone repair. J Bone Joint Surg Am. 1956;38-a(2):270-82.

6. Street JT, McGrath M, O'Regan K, Wakai A, McGuinness A, Redmond HP. Thromboprophylaxis using a low molecular weight heparin delays fracture repair. Clin Orthop Relat Res. 2000;381:278-89.

7. Weitz JI. Low-molecular-weight heparins. N Engl J Med. 1997;337(10):688-98.

8. Lupu C, Poulsen E, Roquefeuil S, Westmuckett AD, Kakkar W, Lupu F. Cellular effects of heparin on the production and release of tissue factor pathway inhibitor in human endothelial cells in culture. Arterioscler Thromb Vasc Biol. 1999;19(9):2251-62.

9. Hak DJ, Stewart RL, Hazelwood SJ. Effect of low molecular weight heparin on fracture healing in a stabilized rat femur fracture model. J Orthop Res. 2006;24(4):645-52.

10. Long A, Zhang L, Zhang $Y$, Jiang B, Mao Z, Li H, et al. Efficacy and safety of rivaroxaban versus low-molecular-weight heparin therapy in patients with lower limb fractures. J Thrombosis Thrombolysis. 2014;38(3):299-305.

11. Gigi R, Salai M, Dolkart O, Chechik O, Katzburg S, Stern N, et al. The effects of direct factor Xa inhibitor (Rivaroxaban) on the human osteoblastic cell line SaOS2. Connect Tissue Res. 2012;53(6):446-50.

12. Bonnarens F, Einhorn TA. Production of a standard closed fracture in laboratory animal bone. J Orthop Res. 1984;2(1):97-101. 
13. Perzborn E, Strassburger J, Wilmen A, Pohlmann J, Roehrig S, Schlemmer $\mathrm{KH}$, et al. In vitro and in vivo studies of the novel antithrombotic agent BAY 59-7939-an oral, direct Factor Xa inhibitor. J Thromb Haemost. 2005:3(3):514-21.

14. Manigrasso MB, O'Connor JP. Characterization of a closed femur fracture model in mice. J Orthop Trauma. 2004;18(10):687-95.

15. Engesaeter $L B$, Ekeland A, Langeland N. Methods for testing the mechanical properties of the rat femur. Acta Orthop Scand. 1978;49(6):512-8.

16. Turner $\mathrm{CH}$, Burr DB. Basic biomechanical measurements of bone: a tutorial. Bone. 1993;14(4):595-608.

17. Sarahrudi K, Kaiser G, Thomas A, Michel M, Wolf H, Mousavi M, et al. The influence of low molecular weight heparin on the expression of osteogenic growth factors in human fracture healing. Int Orthop. 2012:36(5):1095-8.

18. Marsh D. Concepts of fracture union, delayed union, and nonunion. Clin Orthop Relat Res. 1998;355 Suppl:S22-30.

19. Markel MD, Wikenheiser MA, Morin RL, Lewallen DG, Chao EY. Quantification of bone healing: comparison of QCT, SPA, MRI, and DEXA in dog osteotomies. Acta Orthop Scand. 1990;61(6):487-98.

20. Nyman JS, Munoz S, Jadhav S, Mansour A, Yoshii T, Mundy GR, et al. Quantitative measures of femoral fracture repair in rats derived by micro-computed tomography. J Biomech. 2009;42(7):891-7.

21. Tiedeman JJ, Lippiello L, Connolly JF, Strates BS. Quantitative roentgenographic densitometry for assessing fracture healing. Clin Orthop Relat Res. 1990;253:279-86.

22. Gabet Y, Muller R, Regev E, Sela J, Shteyer A, Salisbury K, et al. Osteogenic growth peptide modulates fracture callus structural and mechanical properties. Bone. 2004;35(1):65-73.

\section{Submit your next manuscript to BioMed Central and take full advantage of:}

- Convenient online submission

- Thorough peer review

- No space constraints or color figure charges

- Immediate publication on acceptance

- Inclusion in PubMed, CAS, Scopus and Google Scholar

- Research which is freely available for redistribution 